\title{
Existence Results for Fractional Differential Equations with Separated Boundary Conditions and Fractional Impulsive Conditions
}

\author{
$\mathrm{Xi} \mathrm{Fu}^{1}$ and Xiaoyou Liu $^{2}$ \\ ${ }^{1}$ Department of Mathematics, Shaoxing University, Shaoxing, Zhejiang 312000, China \\ ${ }^{2}$ School of Mathematics and Physics, University of South China, Hengyang, Hunan 421001, China \\ Correspondence should be addressed to Xi Fu; fuxi1984@hotmail.com
}

Received 6 March 2013; Accepted 14 July 2013

Academic Editor: Stanislaw Migorski

Copyright (C) 2013 X. Fu and X. Liu. This is an open access article distributed under the Creative Commons Attribution License, which permits unrestricted use, distribution, and reproduction in any medium, provided the original work is properly cited.

This paper is concerned with the fractional separated boundary value problem of fractional differential equations with fractional impulsive conditions. By means of the Schaefer fixed point theorem, Banach fixed point theorem, and nonlinear alternative of Leray-Schauder type, some existence results are obtained. Examples are given to illustrate the results.

\section{Introduction}

Recently, much attention has been paid to study fractional differential equations due to the fact that they have been proven to be valuable tools in the mathematical modeling of many phenomena in physics, biology, mechanics, and so forth, (see [1-3]).

The theory of impulsive differential equations of integer order has found its extensive applications in realistic mathematical modeling of a wide variety of practical situations and has emerged as an important area of investigation in recent years. For the general theory and applications of impulsive differential equations, see [4-10] and so forth. However, impulsive fractional differential equations have not been much studied, and many aspects of these equations are yet to be explored. For some recent work on impulsive fractional differential equations, we can refer to [11-26] and the references therein.

In this paper, we consider the existence and uniqueness of solutions for the following fractional separated boundary value problem with fractional impulsive conditions:

$$
\begin{array}{r}
{ }^{c} D^{\alpha} x(t)=f(t, x(t)), \\
t \in J:=[0, T], \quad t \neq t_{k}, \quad k=1,2, \ldots, m, \\
\Delta x\left(t_{k}\right)=I_{k}\left(x\left(t_{k}^{-}\right)\right), \quad \Delta\left({ }^{c} D^{\gamma} x\left(t_{k}\right)\right)=I_{k}^{*}\left(x\left(t_{k}^{-}\right)\right), \\
k=1,2, \ldots, m,
\end{array}
$$

$$
\begin{aligned}
& a_{1} x(0)+b_{1}\left({ }^{c} D^{\gamma} x(0)\right)=c_{1}, \\
& a_{2} x(T)+b_{2}\left({ }^{c} D^{\gamma} x(T)\right)=c_{2},
\end{aligned}
$$

where ${ }^{c} D^{\alpha}$ is the Caputo fractional derivative of order $\alpha \epsilon$ $(1,2)$ with the lower limit zero, $0<\gamma<1, f \in C(J \times \mathbb{R}, \mathbb{R})$, $I_{k}, I_{k}^{*} \in C(\mathbb{R}, \mathbb{R}), 0=t_{0}<t_{1}<\cdots<t_{m}<t_{m+1}=T$, $\Delta x\left(t_{k}\right)=x\left(t_{k}^{+}\right)-x\left(t_{k}^{-}\right)$with $x\left(t_{k}^{+}\right)=\lim _{\epsilon \rightarrow 0^{+}} x\left(t_{k}+\epsilon\right), x\left(t_{k}^{-}\right)=$ $\lim _{\epsilon \rightarrow 0^{-}} x\left(t_{k}+\epsilon\right)$ representing the right and left limits of $x(t)$ at $t=t_{k}, \Delta\left({ }^{c} D^{\gamma} x\left(t_{k}\right)\right)$ has a similar meaning for ${ }^{c} D^{\gamma} x\left(t_{k}\right)$, and $a_{i}, b_{i}, c_{i}, i=1,2$, are real constants with $a_{1} \neq 0$ and $a_{2} T^{\gamma} \Gamma(2-$ $\gamma) \neq-b_{2}$.

We note that the papers on this topic cited above except [24] all deal with the Caputo derivative and the impulsive conditions only involve integer order derivatives. Here we study the fractional differential equations with fractional impulsive conditions and fractional separated boundary conditions.

In [24], the author considered the following two impulsive problems:

$$
\begin{array}{r}
{ }^{c} D^{\delta} x(t)=f(t, x(t)), \quad t \in(0,1] \backslash\left\{t_{1}, t_{2}, \ldots, t_{m}\right\}, \\
{ }^{c} D^{\gamma} x\left(t_{k}^{+}\right)-{ }^{c} D^{\gamma} x\left(t_{k}^{-}\right)=J_{k}\left(x\left(t_{k}\right)\right), \\
k=1,2, \ldots, m, \\
x(0)=x_{0}, \quad x^{\prime}(0)=x_{1},
\end{array}
$$


where ${ }^{c} D^{\delta}$ is the Caputo fractional derivative of order $\delta \epsilon$ $(1,2)$ with the lower limit zero, $0<\gamma<1$, and

$$
\begin{gathered}
{ }^{L} D^{\delta} x(t)=f(t, x(t)), \quad t \in(0,1] \backslash\left\{t_{1}, t_{2}, \ldots, t_{m}\right\}, \\
{ }^{L} D^{\gamma} x\left(t_{k}^{+}\right)-{ }^{L} D^{\gamma} x\left(t_{k}^{-}\right)=J_{k}\left(x\left(t_{k}\right)\right), \\
k=1,2, \ldots, m, \\
I^{1-\alpha} x(0)=x_{0},
\end{gathered}
$$

where ${ }^{L} D^{\delta}$ is the Riemann-Liouville fractional derivative of order $\delta \in(0,1)$ with the lower limit zero and $0<\gamma<\delta$.

In [25], Fečkan et al. studied the impulsive problem of the following form:

$$
\begin{gathered}
{ }^{c} D^{\delta} x(t)=f(t, x(t)), \\
t \in(0, T] \backslash\left\{t_{1}, t_{2}, \ldots, t_{m}\right\}, \quad \delta \in(0,1), \\
\Delta x\left(t_{k}\right)=I_{k}\left(x\left(t_{k}^{-}\right)\right), \quad k=1,2, \ldots, m, \\
x(0)=x_{0},
\end{gathered}
$$

where $f:[0, T] \times \mathbb{R} \rightarrow \mathbb{R}$ is jointly continuous, $I_{k}: \mathbb{R} \rightarrow$ $\mathbb{R}$ and $t_{k}$ satisfy $0=t_{0}<t_{1}<\cdots<t_{m+1}=T, x\left(t_{k}^{+}\right)=$ $\lim _{\epsilon \rightarrow 0^{+}} x\left(t_{k}+\epsilon\right)$, and $x\left(t_{k}^{-}\right)=\lim _{\epsilon \rightarrow 0^{-}} x\left(t_{k}+\epsilon\right)$.

Furthermore, Wang et al. [26] considered the impulsive fractional differential equations with boundary conditions as follows:

$$
\begin{gathered}
{ }^{c} D^{\delta} u(t)=h(t), \quad t \in J^{\prime}, \delta \in(1,2), \\
\Delta u\left(t_{k}\right)=y_{k}, \quad \Delta u^{\prime}\left(t_{k}\right)=\bar{y}_{k}, \quad k=1,2, \ldots, m, \\
u(0)=0, \quad u^{\prime}(1)=0,
\end{gathered}
$$

where $y_{k}, \bar{y}_{k} \in \mathbb{R}$.

To the best of our knowledge, there are few papers concerning fractional differential equations with separated boundary conditions $[27,28]$.

The rest of the paper is organized as follows. In Section 2 we introduce some preliminary results needed in the sequel. In Section 3 we present the existence results for the problem (1). Two examples are given in Section 4 to illustrate the results.

\section{Preliminaries}

Let us set $J_{0}=\left[0, t_{1}\right], J_{1}=\left(t_{1}, t_{2}\right], \ldots, J_{m-1}=\left(t_{m-1}, t_{m}\right]$, and $J_{m}=\left(t_{m}, t_{m+1}\right], J^{\prime}:=J \backslash\left\{t_{1}, t_{2}, \ldots, t_{m}\right\}$ and introduce the space $\operatorname{PC}(J, \mathbb{R}):=\left\{u: J \rightarrow \mathbb{R} \mid u \in C\left(J_{k}, \mathbb{R}\right), k=\right.$ $0,1,2, \ldots, m$, and there exist $u\left(t_{k}^{+}\right)$and $u\left(t_{k}^{-}\right), k=1,2, \ldots, m$, with $\left.u\left(t_{k}^{-}\right)=u\left(t_{k}\right)\right\}$. It is clear that $\mathrm{PC}(J, \mathbb{R})$ is a Banach space with the norm $\|u\|=\sup \{|u(t)|: t \in J\}$.
Definition 1 (see [3]). The Riemann-Liouville fractional integral of order $q$ for a continuous function $f:[0, \infty) \rightarrow \mathbb{R}$ is defined as

$$
I^{q} f(t)=\frac{1}{\Gamma(q)} \int_{0}^{t}(t-s)^{q-1} f(s) d s, \quad q>0
$$

which provided that the integral exists.

Definition 2 (see [3]). For $n-1$ times an absolutely continuous function $f:[0, \infty) \rightarrow \mathbb{R}$, the Caputo derivative of order $q$ is defined as

$$
\begin{array}{r}
{ }^{c} D^{q} f(t)=\frac{1}{\Gamma(n-q)} \int_{0}^{t}(t-s)^{n-q-1} f^{(n)}(s) d s, \\
n-1<q<n, \quad n=[q]+1,
\end{array}
$$

where $[q]$ denotes the integer part of the real number $q$.

Lemma 3 (see [3]). Let $\alpha>0$. Then the differential equation

$$
{ }^{c} D^{\alpha} h(t)=0
$$

has solutions $h(t)=c_{0}+c_{1} t+c_{2} t^{2}+\cdots+c_{n-1} t^{n-1}$ and

$$
I^{\alpha c} D^{\alpha} h(t)=h(t)+c_{0}+c_{1} t+c_{2} t^{2}+\cdots+c_{n-1} t^{n-1}
$$

which hold for almost all points on the interval $[0, \infty)$, here $c_{i} \in \mathbb{R}, i=0,1,2, \ldots, n-1, n=[\alpha]+1$.

Definition 4. A function $x \in \mathrm{PC}(J, \mathbb{R})$ with its $\alpha$-derivative existing on $J^{\prime}$ is said to be a solution of the problem (1) if $x$ satisfies the equation ${ }^{c} D^{\alpha} x(t)=f(t, x(t))$ on $J^{\prime}$ and the conditions

$$
\begin{array}{r}
\Delta x\left(t_{k}\right)=I_{k}\left(x\left(t_{k}^{-}\right)\right), \quad \Delta\left({ }^{c} D^{\gamma} x\left(t_{k}\right)\right)=I_{k}^{*}\left(x\left(t_{k}^{-}\right)\right), \\
k=1,2, \ldots, m, \\
a_{1} x(0)+b_{1}\left({ }^{c} D^{\gamma} x(0)\right)=c_{1}, \\
a_{2} x(T)+b_{2}\left({ }^{c} D^{\gamma} x(T)\right)=c_{2}
\end{array}
$$

are satisfied.

By using a similar discussion of [25], we have the following lemma. 
Lemma 5. Let $y \in P C(J, \mathbb{R})$. A function $x$ is a solution of the fractional integral equation:

$$
x(t)=\left\{\begin{array}{l}
\int_{0}^{t} \frac{(t-s)^{\alpha-1}}{\Gamma(\alpha)} y(s) d s+\frac{c_{1}}{a_{1}}-\frac{\Lambda t}{v}-\frac{a_{2} \Pi t}{v}-\frac{a_{2} c_{1} t}{v a_{1}} \\
\quad+\frac{c_{2} t}{v}-\Gamma(2-\gamma) t \sum_{i=1}^{m} \frac{I_{i}^{*}\left(x\left(t_{i}^{-}\right)\right)}{t_{i}^{1-\gamma}}, \quad t \in J_{0} \\
\int_{0}^{t} \frac{(t-s)^{\alpha-1}}{\Gamma(\alpha)} y(s) d s+\frac{c_{1}}{a_{1}}+I_{1}\left(x\left(t_{1}^{-}\right)\right) \\
\quad-\Gamma(2-\gamma) t_{1}^{\gamma} I_{1}^{*}\left(x\left(t_{1}^{-}\right)\right)-\frac{\Lambda t}{v}-\frac{a_{2} \Pi t}{v}-\frac{a_{2} c_{1} t}{v a_{1}} \\
\int_{0}^{t} \frac{(t-s)^{\alpha-1}}{\Gamma(\alpha)} y(s) d s+\frac{c_{1}}{a_{1}}+\sum_{i=1}^{k} I_{i}\left(x\left(t_{i}^{-}\right)\right) \\
-\Gamma(2-\gamma) \sum_{i=1} t_{i}^{\gamma} I_{i}^{*}\left(x\left(t_{i}^{-}\right)\right)-\frac{\Lambda t}{v}-\frac{a_{2} \Pi t}{v}-\frac{a_{2} c_{1} t}{v a_{1}} \\
+\frac{c_{2} t}{v}-\Gamma(2-\gamma) t \sum_{i=k+1}^{m} \frac{I_{i}^{*}\left(x\left(t_{i}^{-}\right)\right)}{t_{i}^{1-\gamma}},
\end{array}\right.
$$

where

$$
\begin{gathered}
v=\frac{a_{2} T \Gamma(2-\gamma)+b_{2} T^{1-\gamma}}{\Gamma(2-\gamma)} \\
\Lambda=a_{2} \int_{0}^{T} \frac{(T-s)^{\alpha-1}}{\Gamma(\alpha)} y(s) d s+b_{2} \int_{0}^{T} \frac{(T-s)^{\alpha-\gamma-1}}{\Gamma(\alpha-\gamma)} y(s) d s \\
\Pi=\sum_{i=1}^{m} I_{i}\left(x\left(t_{i}^{-}\right)\right)-\Gamma(2-\gamma) \sum_{i=1}^{m} t_{i}^{\gamma} I_{i}^{*}\left(x\left(t_{i}^{-}\right)\right)
\end{gathered}
$$

if and only if $x$ is a solution of the impulsive fractional BVP:

$$
\begin{gathered}
{ }^{c} D^{\alpha} x(t)=y(t), \quad t \in J^{\prime}, 1<\alpha<2, \\
\Delta x\left(t_{k}\right)=I_{k}\left(x\left(t_{k}^{-}\right)\right), \quad \Delta\left({ }^{c} D^{\gamma} x\left(t_{k}\right)\right)=I_{k}^{*}\left(x\left(t_{k}^{-}\right)\right), \\
k=1,2, \ldots, m, \\
a_{1} x(0)+b_{1}\left({ }^{c} D^{\gamma} x(0)\right)=c_{1}, \\
a_{2} x(T)+b_{2}\left({ }^{c} D^{\gamma} x(T)\right)=c_{2} .
\end{gathered}
$$

Proof. For $1<\alpha<2$, by Lemma 3, we know that a general solution of the equation ${ }^{c} D^{\alpha} x(t)=y(t)$ on each interval $J_{k}$ $(k=0,1,2, \ldots, m)$ is given by

$$
\begin{aligned}
x(t) & =I^{\alpha} y(t)+d_{k}+e_{k} t \\
& =\int_{0}^{t} \frac{(t-s)^{\alpha-1}}{\Gamma(\alpha)} y(s) d s+d_{k}+e_{k} t, \quad t \in J_{k},
\end{aligned}
$$

where $d_{k}, e_{k} \in \mathbb{R}$ are arbitrary constants. Since ${ }^{c} D^{\gamma} C=0(C$ is a constant), ${ }^{c} D^{\gamma} t=t^{1-\gamma} / \Gamma(2-\gamma)$, and ${ }^{c} D^{\gamma} I^{\alpha} y(t)=I^{\alpha-\gamma} y(t)$ (see [3]), then from (15), we have

$$
\begin{aligned}
{ }^{c} D^{\gamma} x(t) & =I^{\alpha-\gamma} y(t)+\frac{e_{k} t^{1-\gamma}}{\Gamma(2-\gamma)} \\
& =\int_{0}^{t} \frac{(t-s)^{\alpha-\gamma-1}}{\Gamma(\alpha-\gamma)} y(s) d s+\frac{e_{k} t^{1-\gamma}}{\Gamma(2-\gamma)},
\end{aligned}
$$

for $t \in J_{k}$. Applying the boundary conditions of (14), we get

$$
\begin{gathered}
a_{1} \times d_{0}+b_{1} \times 0=c_{1} \\
a_{2} \times\left(\int_{0}^{T} \frac{(T-s)^{\alpha-1}}{\Gamma(\alpha)} y(s) d s+d_{m}+e_{m} T\right) \\
+b_{2} \times\left(\int_{0}^{T} \frac{(T-s)^{\alpha-\gamma-1}}{\Gamma(\alpha-\gamma)} y(s) d s+\frac{e_{m} T^{1-\gamma}}{\Gamma(2-\gamma)}\right)=c_{2} .
\end{gathered}
$$

Next, using the impulsive conditions in (14), we obtain that for $k=1,2, \ldots, m$

$$
\begin{gathered}
d_{k}-d_{k-1}+\left(e_{k}-e_{k-1}\right) t_{k}=I_{k}\left(x\left(t_{k}^{-}\right)\right), \\
\left(e_{k}-e_{k-1}\right) \frac{t_{k}^{1-\gamma}}{\Gamma(2-\gamma)}=I_{k}^{*}\left(x\left(t_{k}^{-}\right)\right) .
\end{gathered}
$$

Now we can derive the values of $d_{k}, e_{k}, k=0,1,2, \ldots, m$ from formulae (17)-(18). That is,

$$
d_{0}=\frac{c_{1}}{a_{1}}
$$

$$
d_{k}=d_{0}+\sum_{i=1}^{k} I_{i}\left(x\left(t_{i}^{-}\right)\right)-\Gamma(2-\gamma) \sum_{i=1}^{k} t_{i}^{\gamma} I_{i}^{*}\left(x\left(t_{i}^{-}\right)\right)
$$

for $k=1,2, \ldots, m$ and

$$
\begin{gathered}
e_{m}=-\frac{1}{v}\left(a_{2} \int_{0}^{T} \frac{(T-s)^{\alpha-1}}{\Gamma(\alpha)} y(s) d s\right. \\
\left.\quad+b_{2} \int_{0}^{T} \frac{(T-s)^{\alpha-\gamma-1}}{\Gamma(\alpha-\gamma)} y(s) d s\right)-\frac{a_{2} d_{m}}{v}+\frac{c_{2}}{v}, \\
e_{k}=e_{m}-\Gamma(2-\gamma) \sum_{i=k+1}^{m} \frac{I_{i}^{*}\left(x\left(t_{i}^{-}\right)\right)}{t_{i}^{1-\gamma}}, \\
\text { for } k=0,1,2, \ldots, m-1 .
\end{gathered}
$$


Hence for $k=0,1,2, \ldots, m$, we have

$$
\begin{aligned}
d_{k}+e_{k} t & \\
= & \frac{c_{1}}{a_{1}}+\sum_{i=1}^{k} I_{i}\left(x\left(t_{i}^{-}\right)\right)-\Gamma(2-\gamma) \sum_{i=1}^{k} t_{i}^{\gamma} I_{i}^{*}\left(x\left(t_{i}^{-}\right)\right)-\frac{t}{v} \\
& \times\left(a_{2} \int_{0}^{T} \frac{(T-s)^{\alpha-1}}{\Gamma(\alpha)} y(s) d s+b_{2} \int_{0}^{T} \frac{(T-s)^{\alpha-\gamma-1}}{\Gamma(\alpha-\gamma)} y(s) d s\right) \\
& -\frac{a_{2} t}{v}\left(\sum_{i=1}^{m} I_{i}\left(x\left(t_{i}^{-}\right)\right)-\Gamma(2-\gamma) \sum_{i=1}^{m} t_{i}^{\gamma} I_{i}^{*}\left(x\left(t_{i}^{-}\right)\right)\right) \\
& -\frac{a_{2} c_{1} t}{v a_{1}}+\frac{c_{2} t}{v}-\Gamma(2-\gamma) t \sum_{i=k+1}^{m} \frac{I_{i}^{*}\left(x\left(t_{i}^{-}\right)\right)}{t_{i}^{1-\gamma} .}
\end{aligned}
$$

Now it is clear that a solution of the problem (14) has the form of (11).

Conversely, assume that $x$ satisfies the fractional integral equation (11). That is, for $t \in J_{k}, k=0,1,2, \ldots, m$, we have

$$
\begin{aligned}
x(t)= & \int_{0}^{t} \frac{(t-s)^{\alpha-1}}{\Gamma(\alpha)} y(s) d s+\frac{c_{1}}{a_{1}}+\sum_{i=1}^{k} I_{i}\left(x\left(t_{i}^{-}\right)\right) \\
& -\Gamma(2-\gamma) \sum_{i=1}^{k} t_{i}^{\gamma} I_{i}^{*}\left(x\left(t_{i}^{-}\right)\right) \\
& -\left(\frac{\Lambda}{v}+\frac{a_{2} \Pi}{v}+\frac{a_{2} c_{1}}{v a_{1}}-\frac{c_{2}}{v}\right) t \\
& -\Gamma(2-\gamma) t \sum_{i=k+1}^{m} \frac{I_{i}^{*}\left(x\left(t_{i}^{-}\right)\right)}{t_{i}^{1-\gamma}} .
\end{aligned}
$$

Since $1<\alpha<2$, we have ${ }^{c} D^{\alpha} C=0$ ( $C$ is a constant) and ${ }^{c} D^{\alpha} t=0$. Using the fact that ${ }^{c} D^{\alpha}$ is the left inverse of $I^{\alpha}$, we get

$$
{ }^{c} D^{\alpha} x(t)=y(t), \quad t \in J^{\prime},
$$

which means that $x$ satisfies the first equation of the impulsive fractional BVP (14). Next we will verify that $x$ satisfies the impulsive conditions. Taking fractional derivative ${ }^{c} D^{\gamma}$ of (22), we have, for $t \in J_{k}$,

$$
\begin{aligned}
{ }^{c} D^{\gamma} x(t)= & \int_{0}^{t} \frac{(t-s)^{\alpha-\gamma-1}}{\Gamma(\alpha-\gamma)} y(s) d s \\
& -\left(\frac{\Lambda}{v}+\frac{a_{2} \Pi}{v}+\frac{a_{2} c_{1}}{v a_{1}}-\frac{c_{2}}{v}\right) \frac{t^{1-\gamma}}{\Gamma(2-\gamma)} \\
& -t^{1-\gamma} \sum_{i=k+1}^{m} \frac{I_{i}^{*}\left(x\left(t_{i}^{-}\right)\right)}{t_{i}^{1-\gamma}} .
\end{aligned}
$$

From (22), we obtain

$$
\begin{aligned}
x\left(t_{k}^{+}\right)= & \int_{0}^{t_{k}} \frac{\left(t_{k}-s\right)^{\alpha-1}}{\Gamma(\alpha)} y(s) d s+\sum_{i=1}^{k} I_{i}\left(x\left(t_{i}^{-}\right)\right) \\
& -\Gamma(2-\gamma) \sum_{i=1}^{k} t_{i}^{\gamma} I_{i}^{*}\left(x\left(t_{i}^{-}\right)\right) \\
& -\left(\frac{\Lambda}{v}+\frac{a_{2} \Pi}{v}+\frac{a_{2} c_{1}}{v a_{1}}-\frac{c_{2}}{v}\right) t_{k} \\
& -\Gamma(2-\gamma) t_{k} \sum_{i=k+1}^{m} \frac{I_{i}^{*}\left(x\left(t_{i}^{-}\right)\right)}{t_{i}^{1-\gamma}}+\frac{c_{1}}{a_{1}}, \\
x\left(t_{k}^{-}\right)= & \int_{0}^{t_{k}} \frac{\left(t_{k}-s\right)^{\alpha-1}}{\Gamma(\alpha)} y(s) d s+\sum_{i=1}^{k-1} I_{i}\left(x\left(t_{i}^{-}\right)\right) \\
& -\Gamma(2-\gamma) \sum_{i=1}^{k-1} t_{i}^{\gamma} I_{i}^{*}\left(x\left(t_{i}^{-}\right)\right) \\
& -\left(\frac{\Lambda}{v}+\frac{a_{2} \Pi}{v}+\frac{a_{2} c_{1}}{v a_{1}}-\frac{c_{2}}{v}\right) t_{k} \\
& -\Gamma(2-\gamma) t_{k} \sum_{i=k}^{m} \frac{I_{i}^{*}\left(x\left(t_{i}^{-}\right)\right)}{t_{i}^{1-\gamma}}+\frac{c_{1}}{a_{1}} .
\end{aligned}
$$

Hence we have, for $k=1,2, \ldots, m$,

$$
\begin{aligned}
\Delta x\left(t_{k}\right)= & I_{k}\left(x\left(t_{k}^{-}\right)\right)-\Gamma(2-\gamma) t_{k}^{\gamma} I_{k}^{*}\left(x\left(t_{k}^{-}\right)\right) \\
& +\Gamma(2-\gamma) t_{k} \frac{I_{k}^{*}\left(x\left(t_{k}^{-}\right)\right)}{t_{k}^{1-\gamma}}=I_{k}\left(x\left(t_{k}^{-}\right)\right) .
\end{aligned}
$$

Similarly, from (24), we can obtain that, for $k=1,2, \ldots, m$,

$$
\Delta\left({ }^{c} D^{\gamma} x\left(t_{k}\right)\right)=t_{k}^{1-\gamma} \frac{I_{k}^{*}\left(x\left(t_{k}^{-}\right)\right)}{t_{k}^{1-\gamma}}=I_{k}^{*}\left(x\left(t_{k}^{-}\right)\right) .
$$

Finally, it follows from (22) and (24) that (since $0 \in J_{0}, T \in$ $\left.J_{m}\right) x(0)=c_{1} / a_{1},{ }^{c} D^{\gamma} x(0)=0$, and

$$
\begin{aligned}
x(T)= & \int_{0}^{T} \frac{(T-s)^{\alpha-1}}{\Gamma(\alpha)} y(s) d s+\frac{c_{1}}{a_{1}} \\
& +\Pi-\left(\frac{\Lambda}{v}+\frac{a_{2} \Pi}{v}+\frac{a_{2} c_{1}}{v a_{1}}-\frac{c_{2}}{v}\right) T, \\
{ }^{c} D^{\gamma} x(T)= & \int_{0}^{T} \frac{(T-s)^{\alpha-\gamma-1}}{\Gamma(\alpha-\gamma)} y(s) d s \\
& -\left(\frac{\Lambda}{v}+\frac{a_{2} \Pi}{v}+\frac{a_{2} c_{1}}{v a_{1}}-\frac{c_{2}}{v}\right) \frac{T^{1-\gamma}}{\Gamma(2-\gamma)} .
\end{aligned}
$$


Now we get

$$
\begin{gathered}
a_{1} x(0)+b_{1}\left({ }^{c} D^{\gamma} x(0)\right)=c_{1}, \\
a_{2} x(T)+b_{2}\left({ }^{c} D^{\gamma} x(T)\right) \\
=\Lambda+\frac{a_{2} c_{1}}{a_{1}}+a_{2} \Pi-a_{2} T\left(\frac{\Lambda}{v}+\frac{a_{2} \Pi}{v}+\frac{a_{2} c_{1}}{v a_{1}}-\frac{c_{2}}{v}\right) \\
-\frac{b_{2} T^{1-\gamma}}{\Gamma(2-\gamma)}\left(\frac{\Lambda}{v}+\frac{a_{2} \Pi}{v}+\frac{a_{2} c_{1}}{v a_{1}}-\frac{c_{2}}{v}\right)=c_{2} .
\end{gathered}
$$

Therefore $x$ given by (11) satisfies the impulsive fractional boundary value problem (14). The proof is complete.

Remark 6. We notice that the expression of (11) does not depend on the parameter $b_{1}$ appearing in the boundary conditions of the problem (14). Thus by Lemma 5, we conclude that the parameter $b_{1}$ is of arbitrary nature of the problem (14).

Let $X, Y$ be Banach spaces and $f: X \rightarrow Y$, and we say that $f$ is a compact if the image of each bounded set in $X$ under $f$ is relatively compact. The following are two fixed point theorems which will be used in the sequel.

Theorem 7 (nonlinear alternative of Leray-Schauder type [29]). Let $X$ be a Banach space, $C$ a nonempty convex subset of $X$, and $U$ a nonempty open subset of $C$ with $0 \in U$. Suppose that $P: \bar{U} \rightarrow C$ is a continuous and compact map. Then either (a) $P$ has a fixed point in $\bar{U}$ or $(b)$ there exist a $x \in \partial U$ (the boundary of $U$ ) and $\lambda \in(0,1)$ with $x=\lambda P(x)$.

Theorem 8 (Schaefer fixed point theorem [30]). Let $X$ be a normed space and $P$ a continuous mapping of $X$ into $X$ which is compact on each bounded subset $B$ of $X$. Then either (I) the equation $x=\lambda P x$ has a solution for $\lambda=1$ or (II) the set of all such solutions $x$, for $0<\lambda<1$, is unbounded.

\section{Main Results}

This section deals with the existence and uniqueness of solutions for the problem (1).

In view of Lemma 5, we define an operator $F: \operatorname{PC}(J$, $\mathbb{R}) \rightarrow \operatorname{PC}(J, \mathbb{R})$ by

$$
\begin{aligned}
&(F x)(t)= \int_{0}^{t} \frac{(t-s)^{\alpha-1}}{\Gamma(\alpha)} f(s, x(s)) d s+\frac{c_{1}}{a_{1}} \\
&+\sum_{i=1}^{k} I_{i}\left(x\left(t_{i}^{-}\right)\right)-\Gamma(2-\gamma) \sum_{i=1}^{k} t_{i}^{\gamma} I_{i}^{*}\left(x\left(t_{i}^{-}\right)\right) \\
&-\frac{\Lambda_{x} t}{v}-\frac{a_{2} \Pi_{x} t}{v}-\frac{a_{2} c_{1} t}{v a_{1}}+\frac{c_{2} t}{v} \\
&-\Gamma(2-\gamma) t \sum_{i=k+1}^{m} \frac{I_{i}^{*}\left(x\left(t_{i}^{-}\right)\right)}{t_{i}^{1-\gamma}}, \quad t \in J_{k}, \\
& k=0,1,2, \ldots, m,
\end{aligned}
$$

with

$$
\begin{gathered}
\Lambda_{x}=a_{2} \int_{0}^{T} \frac{(T-s)^{\alpha-1}}{\Gamma(\alpha)} f(s, x(s)) d s+b_{2} \\
\times \int_{0}^{T} \frac{(T-s)^{\alpha-\gamma-1}}{\Gamma(\alpha-\gamma)} f(s, x(s)) d s, \\
\Pi_{x}=\sum_{i=1}^{m} I_{i}\left(x\left(t_{i}^{-}\right)\right)-\Gamma(2-\gamma) \sum_{i=1}^{m} t_{i}^{\gamma} I_{i}^{*}\left(x\left(t_{i}^{-}\right)\right) .
\end{gathered}
$$

Here $\Lambda_{x}, \Pi_{x}$ mean that $\Lambda, \Pi$ defined in Lemma 5 are related to $x \in \mathrm{PC}(J, \mathbb{R})$. It is obvious that $F$ is well defined because of the continuity of $f, I_{k}$, and $I_{k}^{*}$. Observe that the problem (1) has solutions if and only if the operator equation $F x=x$ has fixed points.

Lemma 9. The operator $F: P C(J, \mathbb{R}) \rightarrow P C(J, \mathbb{R})$ defined by (31) is completely continuous.

Proof. Since $f, I_{k}$, and $I_{k}^{*}$ are continuous, it is easy to show that $F$ is continuous on $\mathrm{PC}(J, \mathbb{R})$.

Let $B \subseteq \mathrm{PC}(J, \mathbb{R})$ be bounded. Then there exist positive constants $N_{i}, i=1,2,3$, such that $|f(t, x(t))| \leq N_{1}$, $\left|I_{k}\left(x\left(t_{k}^{-}\right)\right)\right| \leq N_{2}$, and $\left|I_{k}^{*}\left(x\left(t_{k}^{-}\right)\right)\right| \leq N_{3}$ for all $t \in J, x \in B$, $k=1,2, \ldots, m$. Thus, for $x \in B$ and $t \in J$, we have

$$
\begin{aligned}
|(F x)(t)| \leq & \frac{N_{1} T^{\alpha}}{\Gamma(\alpha+1)}+\frac{\left|c_{1}\right|}{\left|a_{1}\right|}+m N_{2} \\
& +\Gamma(2-\gamma) N_{3} \sum_{i=1}^{m} t_{i}^{\gamma}+\frac{\left|\Lambda_{x}\right| T}{|v|} \\
& +\frac{\left|a_{2} \Pi_{x}\right| T}{|v|}+\frac{\left|a_{2} c_{1}\right| T}{\left|v a_{1}\right|}+\frac{\left|c_{2}\right| T}{|v|} \\
& +\Gamma(2-\gamma) T N_{3} \sum_{i=1}^{m} t_{i}^{\gamma-1}, \\
\left|\Lambda_{x}\right| \leq & \frac{\left|a_{2}\right| N_{1} T^{\alpha}}{\Gamma(\alpha+1)}+\frac{\left|b_{2}\right| N_{1} T^{\alpha-\gamma}}{\Gamma(\alpha-\gamma+1)}, \\
\left|\Pi_{x}\right| \leq & m N_{2}+\Gamma(2-\gamma) N_{3} \sum_{i=1}^{m} t_{i}^{\gamma} .
\end{aligned}
$$

Now we can obtain that, for all $x \in B$, and $t \in J$,

$$
\begin{aligned}
|(F x)(t)| & \leq \frac{N_{1} T^{\alpha}}{\Gamma(\alpha+1)}+\frac{T}{|v|}\left(\frac{\left|a_{2}\right| N_{1} T^{\alpha}}{\Gamma(\alpha+1)}+\frac{\left|b_{2}\right| N_{1} T^{\alpha-\gamma}}{\Gamma(\alpha-\gamma+1)}\right) \\
& +\left(1+\frac{\left|a_{2}\right| T}{|v|}\right) m N_{2}+\Gamma(2-\gamma) N_{3} \\
& \times\left(\left(1+\frac{\left|a_{2}\right| T}{|v|}\right) \sum_{i=1}^{m} t_{i}^{\gamma}+T \sum_{i=1}^{m} t_{i}^{\gamma-1}\right) \\
& +\frac{\left|a_{2} c_{1}\right| T}{\left|v a_{1}\right|}+\frac{\left|c_{2}\right| T}{|v|}+\frac{\left|c_{1}\right|}{\left|a_{1}\right|},
\end{aligned}
$$


which implies that the operator $F$ is uniformly bounded on $B$.

On the other hand, let $x \in B$ and for any $\tau_{1} \cdot \tau_{2} \in J_{k}$, $k=0,1,2, \ldots, m$, with $\tau_{1}<\tau_{2}$, we have

$$
\begin{aligned}
&\left|(F x)\left(\tau_{2}\right)-(F x)\left(\tau_{1}\right)\right| \\
& \leq \mid \int_{0}^{\tau_{2}} \frac{\left(\tau_{2}-s\right)^{\alpha-1}}{\Gamma(\alpha)} f(s, x(s)) d s \\
& \quad-\int_{0}^{\tau_{1}} \frac{\left(\tau_{1}-s\right)^{\alpha-1}}{\Gamma(\alpha)} f(s, x(s)) d s \mid \\
& \quad+\left(\frac{\left|\Lambda_{x}\right|}{|v|}+\frac{\left|a_{2} \Pi_{x}\right|}{|v|}+\frac{\left|a_{2} c_{1}\right|}{\left|v a_{1}\right|}+\frac{\left|c_{2}\right|}{|v|}\right)\left(\tau_{2}-\tau_{1}\right) \\
& \quad+\Gamma(2-\gamma) \sum_{i=k+1}^{m} t_{i}^{\gamma-1}\left|I_{i}^{*}\left(x\left(t_{i}^{-}\right)\right)\right|\left(\tau_{2}-\tau_{1}\right) \\
& \leq \frac{N_{1}\left(\tau_{2}^{\alpha}-\tau_{1}^{\alpha}\right)}{\Gamma(\alpha+1)}+\left(\frac{\left|\Lambda_{x}\right|}{|v|}+\frac{\left|a_{2} \Pi_{x}\right|}{|v|}+\frac{\left|a_{2} c_{1}\right|}{\left|v a_{1}\right|}+\frac{\left|c_{2}\right|}{|v|}\right) \\
& \times\left(\tau_{2}-\tau_{1}\right)+\Gamma(2-\gamma) N_{3} \sum_{i=k+1}^{m} t_{i}^{\gamma-1}\left(\tau_{2}-\tau_{1}\right) .
\end{aligned}
$$

By (34) and the above inequality, we deduce that

$$
\left|(F x)\left(\tau_{2}\right)-(F x)\left(\tau_{1}\right)\right| \longrightarrow 0 \quad \text { as } \tau_{2} \longrightarrow \tau_{1} .
$$

This implies that $F$ is equicontinuous on the interval $J_{k}$. Hence by PC-type Arzela-Ascoli theorem (see Theorem 2.1 [10]), the operator $F: \mathrm{PC}(J, \mathbb{R}) \rightarrow \mathrm{PC}(J, \mathbb{R})$ is completely continuous.

Theorem 10. Assume that (1) there exist $h \in L^{\infty}\left(J, \mathbb{R}^{+}\right)$and $\varphi:[0, \infty) \rightarrow(0, \infty)$ continuous, nondecreasing such that $|f(t, x)| \leq h(t) \varphi(|x|)$ for $(t, x) \in J \times \mathbb{R}$; (2) there exist $\psi, \psi^{*}:[0, \infty) \rightarrow(0, \infty)$ continuous, nondecreasing such that $\left|I_{k}(x)\right| \leq \psi(|x|),\left|I_{k}^{*}(x)\right| \leq \psi^{*}(|x|)$ for all $x \in \mathbb{R}$ and $k=1,2, \ldots, m$; (3) there exists a constant $M>0$ such that

$$
\frac{M}{P \varphi(M)\|h\|_{L^{\infty}}+Q \psi(M)+R \psi^{*}(M)+H}>1,
$$

where

$$
\begin{gathered}
P=\frac{T^{\alpha}}{\Gamma(\alpha+1)}+\frac{T}{|v|}\left(\frac{\left|a_{2}\right| T^{\alpha}}{\Gamma(\alpha+1)}+\frac{\left|b_{2}\right| T^{\alpha-\gamma}}{\Gamma(\alpha-\gamma+1)}\right), \\
Q=m\left(1+\frac{\left|a_{2}\right| T}{|v|}\right), \\
R=\Gamma(2-\gamma)\left[\left(1+\frac{\left|a_{2}\right| T}{|v|}\right) \sum_{i=1}^{m} t_{i}^{\gamma}+T \sum_{i=1}^{m} t_{i}^{\gamma-1}\right], \\
H=\frac{\left|a_{2} c_{1}\right| T}{\left|v a_{1}\right|}+\frac{\left|c_{2}\right| T}{|v|}+\frac{\left|c_{1}\right|}{\left|a_{1}\right|} .
\end{gathered}
$$

Then, BVP (1) has at least one solution.
Proof. We will show that the operator $F$ defined by (31) satisfies the assumptions of the nonlinear alternative of LeraySchauder type.

From Lemma 9, the operator $F: \mathrm{PC}(J, \mathbb{R}) \rightarrow \mathrm{PC}(J, \mathbb{R})$ is continuous and completely continuous.

Let $x \in \mathrm{PC}(J, \mathbb{R})$ such that $x(t)=\lambda(F x)(t)$ for some $\lambda \epsilon$ $(0,1)$. Then using the computations in proving that $F$ maps bounded sets into bounded sets in Lemma 9, we have

$$
\begin{aligned}
|x(t)| \leq & \|h\|_{L^{\infty}} \varphi(\|x\|) \\
& \times\left[\frac{T^{\alpha}}{\Gamma(\alpha+1)}+\frac{T}{|v|}\left(\frac{\left|a_{2}\right| T^{\alpha}}{\Gamma(\alpha+1)}+\frac{\left|b_{2}\right| T^{\alpha-\gamma}}{\Gamma(\alpha-\gamma+1)}\right)\right] \\
& +\Gamma(2-\gamma) \psi^{*}(\|x\|)\left(\left(1+\frac{\left|a_{2}\right| T}{|v|}\right) \sum_{i=1}^{m} t_{i}^{\gamma}+T \sum_{i=1}^{m} t_{i}^{\gamma-1}\right) \\
& +\left(1+\frac{\left|a_{2}\right| T}{|v|}\right) m \psi(\|x\|)+\frac{\left|a_{2} c_{1}\right| T}{\left|v a_{1}\right|}+\frac{\left|c_{2}\right| T}{|v|}+\frac{\left|c_{1}\right|}{\left|a_{1}\right|} .
\end{aligned}
$$

Consequently, we have

$$
\frac{\|x\|}{P\|h\|_{L^{\infty}} \varphi(\|x\|)+Q \psi(\|x\|)+R \psi^{*}(\|x\|)+H} \leq 1 .
$$

Then by condition (38), $\|x\| \neq M$. Let us set

$$
U=\{x \in \operatorname{PC}(J, \mathbb{R}):\|x\|<M\} .
$$

The operator $F: \bar{U} \rightarrow \operatorname{PC}(J, \mathbb{R})$ is continuous and compact. From the choice of the set $U$, there is no $x \in$ $\partial U$ such that $x=\lambda F x$ for some $\lambda \in(0,1)$. Therefore by the nonlinear alternative of Leray-Schauder type (see Theorem 7), we deduce that $F$ has a fixed point $x$ in $\bar{U}$ which is a solution of the problem (1). The proof is complete.

Theorem 11. Assume that there exist $h \in L^{\infty}\left(J, \mathbb{R}^{+}\right)$and positive constants $H_{1}, H_{2}$ such that, for $t \in J, x \in \mathbb{R}, k=$ $1,2, \ldots, m$,

$$
|f(t, x)| \leq h(t), \quad\left|I_{k}(x)\right| \leq H_{1}, \quad\left|I_{k}^{*}(x)\right| \leq H_{2} .
$$

Then, BVP (1) has at least one solution on $[0, T]$.

Proof. Lemma 9 tells us that the operator $F: \operatorname{PC}(J, \mathbb{R}) \rightarrow$ $\mathrm{PC}(J, \mathbb{R})$ defined by (31) is continuous and compact on each bounded subset $B$ of $P C(J, \mathbb{R})$. 
Let $V=\{u \in \operatorname{PC}(J, \mathbb{R}): u=\lambda F u, 0<\lambda<1\}$. Since, for each $t \in J$,

$$
\begin{aligned}
|x(t)| & \\
= & |\lambda(F x)(t)| \\
\leq & \|h\|_{L^{\infty}} \\
& \times\left[\frac{T^{\alpha}}{\Gamma(\alpha+1)}+\frac{T}{|v|}\left(\frac{\left|a_{2}\right| T^{\alpha}}{\Gamma(\alpha+1)}+\frac{\left|b_{2}\right| T^{\alpha-\gamma}}{\Gamma(\alpha-\gamma+1)}\right)\right] \\
& +\Gamma(2-\gamma) H_{2}\left(\left(1+\frac{\left|a_{2}\right| T}{|v|}\right) \sum_{i=1}^{m} t_{i}^{\gamma}+T \sum_{i=1}^{m} t_{i}^{\gamma-1}\right) \\
& +\left(1+\frac{\left|a_{2}\right| T}{|v|}\right) m H_{1}+\frac{\left|a_{2} c_{1}\right| T}{\left|v a_{1}\right|}+\frac{\left|c_{2}\right| T}{|v|}+\frac{\left|c_{1}\right|}{\left|a_{1}\right|}
\end{aligned}
$$

we know that $V$ is bounded. Thus, by Theorem 8 , the operator $F$ has at least one fixed point. Hence the problem (1) has at least one solution. The proof is completed.

Theorem 12. Assume that there exist $h \in L^{\infty}\left(J, \mathbb{R}^{+}\right)$and positive constants $L, L^{*}$ such that, for $t \in J, x, y \in \mathbb{R}, k=$ $1,2, \ldots, m$,

$$
\begin{gathered}
|f(t, x)-f(t, y)| \leq h(t)|x-y|, \\
\left|I_{k}(x)-I_{k}(y)\right| \leq L|x-y|, \\
\left|I_{k}^{*}(x)-I_{k}^{*}(y)\right| \leq L^{*}|x-y| .
\end{gathered}
$$

\section{Moreover}

$$
\begin{gathered}
\frac{\|h\|_{L^{\infty}} T^{\alpha}}{\Gamma(\alpha+1)}+\frac{T\|h\|_{L^{\infty}}}{|v|}\left(\frac{\left|a_{2}\right| T^{\alpha}}{\Gamma(\alpha+1)}+\frac{\left|b_{2}\right| T^{\alpha-\gamma}}{\Gamma(\alpha-\gamma+1)}\right) \\
+\left(1+\frac{\left|a_{2}\right| T}{|v|}\right) m L+\Gamma(2-\gamma) L^{*} \\
\times\left[\left(1+\frac{\left|a_{2}\right| T}{|v|}\right) \sum_{i=1}^{m} t_{i}^{\gamma}+T \sum_{i=1}^{m} t_{i}^{\gamma-1}\right]<1 .
\end{gathered}
$$

Then, BVP (1) has a unique solution on J.

Proof. Let $x, y \in \operatorname{PC}(J, \mathbb{R})$. Then for each $t \in J$, we have

$$
\begin{aligned}
& |(F x)(t)-(F y)(t)| \\
& \leq \int_{0}^{t} \frac{(t-s)^{\alpha-1}}{\Gamma(\alpha)}|f(s, x(s))-f(s, y(s))| d s \\
& \quad+\sum_{i=1}^{m}\left|I_{i}\left(x\left(t_{i}^{-}\right)\right)-I_{i}\left(y\left(t_{i}^{-}\right)\right)\right|
\end{aligned}
$$

$$
\begin{aligned}
& +\Gamma(2-\gamma) \sum_{i=1}^{m} t_{i}^{\gamma}\left|I_{i}^{*}\left(x\left(t_{i}^{-}\right)\right)-I_{i}^{*}\left(y\left(t_{i}^{-}\right)\right)\right| \\
& +\frac{T}{|v|}\left|\Lambda_{x}-\Lambda_{y}\right|+\frac{\left|a_{2}\right| T}{|v|}\left|\Pi_{x}-\Pi_{y}\right|+\Gamma(2-\gamma) T \\
& \times \sum_{i=1}^{m} t_{i}^{\gamma-1}\left|I_{i}^{*}\left(x\left(t_{i}^{-}\right)\right)-I_{i}^{*}\left(y\left(t_{i}^{-}\right)\right)\right| .
\end{aligned}
$$

Since

$$
\begin{gathered}
\left|\Lambda_{x}-\Lambda_{y}\right| \\
\leq\left|a_{2}\right| \int_{0}^{T} \frac{(T-s)^{\alpha-1}}{\Gamma(\alpha)}|f(s, x(s))-f(s, y(s))| d s \\
\quad+\left|b_{2}\right| \int_{0}^{T} \frac{(T-s)^{\alpha-\gamma-1}}{\Gamma(\alpha-\gamma)}|f(s, x(s))-f(s, y(s))| d s \\
\leq \frac{\|h\|_{L^{\infty}}\left|a_{2}\right| T^{\alpha}}{\Gamma(\alpha+1)}\|x-y\|+\frac{\|h\|_{L^{\infty}}\left|b_{2}\right| T^{\alpha-\gamma}}{\Gamma(\alpha-\gamma+1)}\|x-y\|, \\
\left|\Pi_{x}-\Pi_{y}\right| \leq \sum_{i=1}^{m}\left|I_{i}\left(x\left(t_{i}^{-}\right)\right)-I_{i}\left(y\left(t_{i}^{-}\right)\right)\right| \\
\quad+\Gamma(2-\gamma) \sum_{i=1}^{m} t_{i}^{\gamma}\left|I_{i}^{*}\left(x\left(t_{i}^{-}\right)\right)-I_{i}^{*}\left(y\left(t_{i}^{-}\right)\right)\right| \\
\leq m L\|x-y\|+\Gamma(2-\gamma) L^{*} \sum_{i=1}^{m} t_{i}^{\gamma}\|x-y\|,
\end{gathered}
$$

then combining these two estimations with (47), we obtain

$$
\begin{aligned}
\|F x-F y\| & \\
\leq[ & \frac{T\|h\|_{L^{\infty}}}{|v|}\left(\frac{\left|a_{2}\right| T^{\alpha}}{\Gamma(\alpha+1)}+\frac{\left|b_{2}\right| T^{\alpha-\gamma}}{\Gamma(\alpha-\gamma+1)}\right) \\
& +\Gamma(2-\gamma) L^{*}\left(\left(1+\frac{\left|a_{2}\right| T}{|v|}\right) \sum_{i=1}^{m} t_{i}^{\gamma}+T \sum_{i=1}^{m} t_{i}^{\gamma-1}\right) \\
& \left.+\frac{\|h\|_{L^{\infty}} T^{\alpha}}{\Gamma(\alpha+1)}+\left(1+\frac{\left|a_{2}\right| T}{|v|}\right) m L\right]\|x-y\| .
\end{aligned}
$$

Therefore, by (46), the operator $F$ is a contraction mapping on $\operatorname{PC}(J, \mathbb{R})$. Then it follows Banach's fixed point theorem that the problem (1) has a unique solution on $J$. This completes the proof.

\section{Examples}

Finally we give two simple examples to show the applicability of our results. 
Example 1. Consider the following impulsive fractional separated BVP:

$$
\begin{gathered}
{ }^{c} D^{7 / 4} x(t)=\frac{\cos t}{(t+6)^{2}}(x(t)+\arctan x(t)), \\
t \in[0,1], \quad t \neq \frac{1}{2}, \\
\Delta x\left(\frac{1}{2}\right)=\frac{\left|x\left((1 / 2)^{-}\right)\right|}{\left(17+\left|x\left((1 / 2)^{-}\right)\right|\right)}, \\
\Delta\left({ }^{c} D^{1 / 4} x\left(\frac{1}{2}\right)\right)=\frac{\left|x\left((1 / 2)^{-}\right)\right|}{(20+|x((1 / 2))|)} \\
x(0)+2\left({ }^{c} D^{1 / 4} x(0)\right)=\frac{1}{2}, \\
\frac{1}{2} x(1)+\frac{1}{3}\left({ }^{c} D^{1 / 4} x(1)\right)=2 .
\end{gathered}
$$

Here $\alpha=7 / 4, \gamma=1 / 4, T=1$, and $m=1$. Clearly, we can take $h(t)=2 \cos t /(t+6)^{2}, L=1 / 17$ and $L^{*}=1 / 20$ such that the relations (45) hold. Moreover

$$
\begin{aligned}
& \frac{\|h\|_{L^{\infty}} T^{\alpha}}{\Gamma(\alpha+1)}+\frac{T\|h\|_{L^{\infty}}}{|v|}\left(\frac{\left|a_{2}\right| T^{\alpha}}{\Gamma(\alpha+1)}+\frac{\left|b_{2}\right| T^{\alpha-\gamma}}{\Gamma(\alpha-\gamma+1)}\right) \\
& +\left(1+\frac{\left|a_{2}\right| T}{|v|}\right) m L+\Gamma(2-\gamma) L^{*} \\
& \quad \times\left[\left(1+\frac{\left|a_{2}\right| T}{|v|}\right) \sum_{i=1}^{m} t_{i}^{\gamma}+T \sum_{i=1}^{m} \frac{1}{t_{i}^{1-\gamma}}\right] \\
& \quad \approx \frac{1}{18} \times 1.2728+\frac{1}{17} \times 1.5796+\frac{1}{20} \times 2.7666<1 .
\end{aligned}
$$

Thus, all the assumptions of Theorem 12 are satisfied. Hence, by the conclusion of Theorem 12, the impulsive fractional $\operatorname{BVP}(50)$ has a unique solution on $[0,1]$.

Example 2. Consider the following impulsive fractional separated BVP:

$$
\begin{aligned}
& { }^{c} D^{3 / 2} x(t)=5 t^{2}+e^{-|x(t)|}+\sin x(t), \\
& t \in[0,1], \quad t \neq \frac{1}{4}, \\
& \Delta x\left(\frac{1}{4}\right)=\frac{2\left|x\left((1 / 4)^{-}\right)\right|}{\left(1+\left|x\left((1 / 4)^{-}\right)\right|\right)}, \\
& \Delta\left({ }^{c} D^{1 / 2} x\left(\frac{1}{4}\right)\right)=\cos x\left(\frac{1^{-}}{4}\right)+3, \\
& 3 x(0)+\frac{1}{2}\left({ }^{c} D^{1 / 2} x(0)\right)=1, \\
& 2 x(1)+3\left({ }^{c} D^{1 / 2} x(1)\right)=2.5 .
\end{aligned}
$$

In the context of this problem, we have

$$
\begin{gathered}
|f(t, x)|=\left|5 t^{2}+e^{-|x|}+\sin x\right| \leq 7, \quad t \in[0,1], \quad x \in \mathbb{R}, \\
\left|I_{k}(x)\right| \leq 2, \quad\left|I_{k}^{*}(x)\right| \leq 4, \quad x \in \mathbb{R} .
\end{gathered}
$$

Put $h(t) \equiv 7, H_{1}=2$, and $H_{2}=4$. Then from Theorem 11, the impulsive fractional BVP (52) has at least one solution on $[0,1]$.

\section{References}

[1] J. Sabatier, O. P. Agrawal, and J. A. T. Machado, Eds., Advances in Fractional Calculus: Theoretical Developments and Applications in Physics and Engineering, Springer, Dordrecht, The Netherlands, 2007.

[2] V. Lakshmikantham, S. Leela, and J. V. Devi, Theory of Fractional Dynamic Systems, Cambridge Scientific Publishers, 2009.

[3] A. A. Kilbas, H. M. Srivastava, and J. J. Trujillo, Theory and Applications of Fractional Differential Equations, vol. 204 of North-Holland Mathematics Studies, Elsevier Science B.V., Amsterdam, The Netherlands, 2006.

[4] V. Lakshmikantham, D. D. Baĭnov, and P. S. Simeonov, Theory of Impulsive Differential Equations, vol. 6 of Series in Modern Applied Mathematics, World Scientific, Singapore, 1989.

[5] Z. Luo and J. J. Nieto, "New results for the periodic boundary value problem for impulsive integro-differential equations," Nonlinear Analysis: Theory, Methods \& Applications, vol. 70, no. 6, pp. 2248-2260, 2009.

[6] Z. Luo and J. Shen, "Stability of impulsive functional differential equations via the Liapunov functional," Applied Mathematics Letters, vol. 22, no. 2, pp. 163-169, 2009.

[7] Z. Liu and J. Liang, "A class of boundary value problems for firstorder impulsive integro-differential equations with deviating arguments," Journal of Computational and Applied Mathematics, vol. 237, no. 1, pp. 477-486, 2013.

[8] Z. He and J. Yu, "Periodic boundary value problem for firstorder impulsive ordinary differential equations," Journal of Mathematical Analysis and Applications, vol. 272, no. 1, pp. 6778, 2002.

[9] L. Zhang, "Boundary value problem for first order impulsive functional integro-differential equations," Journal of Computational and Applied Mathematics, vol. 235, no. 8, pp. 2442-2450, 2011.

[10] W. Wei, X. Xiang, and Y. Peng, "Nonlinear impulsive integrodifferential equations of mixed type and optimal controls," Optimization, vol. 55, no. 1-2, pp. 141-156, 2006.

[11] H. Wang, "Existence results for fractional functional differential equations with impulses," Journal of Applied Mathematics and Computing, vol. 38, no. 1-2, pp. 85-101, 2012.

[12] M. Benchohra, S. Hamani, J. J. Nieto, and B. A. Slimani, "Existence of solutions to differential inclusions with fractional order and impulses," Electronic Journal of Differential Equations, vol. 2010, no. 80, pp. 1-18, 2010.

[13] B. Ahmad and S. Sivasundaram, "Existence results for nonlinear impulsive hybrid boundary value problems involving fractional differential equations," Nonlinear Analysis: Hybrid Systems, vol. 3, no. 3, pp. 251-258, 2009.

[14] B. Ahmad and S. Sivasundaram, "Existence of solutions for impulsive integral boundary value problems of fractional 
order," Nonlinear Analysis: Hybrid Systems, vol. 4, no. 1, pp. 134141, 2010.

[15] Z. Liu and X. Li, "On the controllability of impulsive fractional evolution inclusions in Banach spaces," Journal of Optimization Theory and Applications, vol. 156, no. 1, pp. 167-182, 2013.

[16] Y. Tian and Z. Bai, "Existence results for the three-point impulsive boundary value problem involving fractional differential equations," Computers \& Mathematics with Applications, vol. 59, no. 8, pp. 2601-2609, 2010.

[17] J. Cao and H. Chen, "Some results on impulsive boundary value problem for fractional differential inclusions," Electronic Journal of Qualitative Theory of Differential Equations, vol. 2011, no. 11, pp. 1-24, 2011.

[18] G. Wang, B. Ahmad, and L. Zhang, "Impulsive anti-periodic boundary value problem for nonlinear differential equations of fractional order," Nonlinear Analysis: Theory, Methods \& Applications, vol. 74, no. 3, pp. 792-804, 2011.

[19] T. L. Guo and J. Wei, "Impulsive problems for fractional differential equations with boundary value conditions," Computers \& Mathematics with Applications, vol. 64, no. 10, pp. 3281-3291, 2012.

[20] G. Wang, B. Ahmad, and L. Zhang, "Some existence results for impulsive nonlinear fractional differential equations with mixed boundary conditions," Computers \& Mathematics with Applications, vol. 62, no. 3, pp. 1389-1397, 2011.

[21] Z. Tai and S. Lun, "On controllability of fractional impulsive neutral infinite delay evolution integrodifferential systems in Banach spaces," Applied Mathematics Letters, vol. 25, no. 2, pp. 104-110, 2012.

[22] X. Wang, "Existence of solutions for nonlinear impulsive higher order fractional differential equations," Electronic Journal of Qualitative Theory of Differential Equations, vol. 2011, no. 80, pp. $1-12,2011$.

[23] C. Bai, "Existence result for boundary value problem of nonlinear impulsive fractional differential equation at resonance," Journal of Applied Mathematics and Computing, vol. 39, no. 1-2, pp. 421-443, 2012.

[24] N. Kosmatov, "Initial value problems of fractional order with fractional impulsive conditions," Results in Mathematics, vol. 63, no. 3-4, pp. 1289-1310, 2013.

[25] M. Fečkan, Y. Zhou, and J. R. Wang, "On the concept and existence of solution for impulsive fractional differential equations," Communications in Nonlinear Science and Numerical Simulation, vol. 17, no. 7, pp. 3050-3060, 2012.

[26] J. R. Wang, Y. Zhou, and M. Fečkan, “On recent developments in the theory of boundary value problems for impulsive fractional differential equations," Computers \& Mathematics with Applications, vol. 64, no. 10, pp. 3008-3020, 2012.

[27] B. Ahmad and S. K. Ntouyas, "A note on fractional differential equations with fractional separated boundary conditions," Abstract and Applied Analysis, vol. 2012, Article ID 818703, 11 pages, 2012.

[28] B. Ahmad and S. K. Ntouyas, "Fractional differential inclusions with fractional separated boundary conditions," Fractional Calculus and Applied Analysis, vol. 15, no. 3, pp. 362-382, 2012.

[29] A. Granas and J. Dugundji, Fixed Point Theory, Springer Monographs in Mathematics, Springer, New York, NY, USA, 2003.

[30] D. R. Smart, Fixed Point Theorems, Cambridge University Press, London, UK, 1974. 


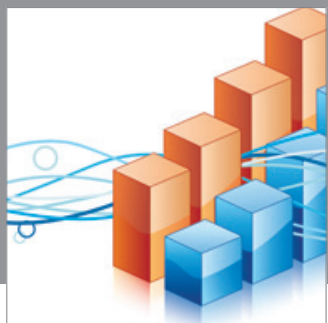

Advances in

Operations Research

mansans

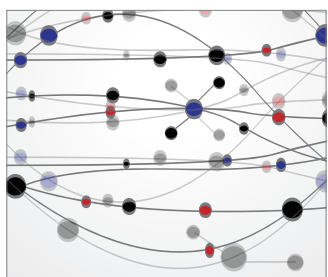

The Scientific World Journal
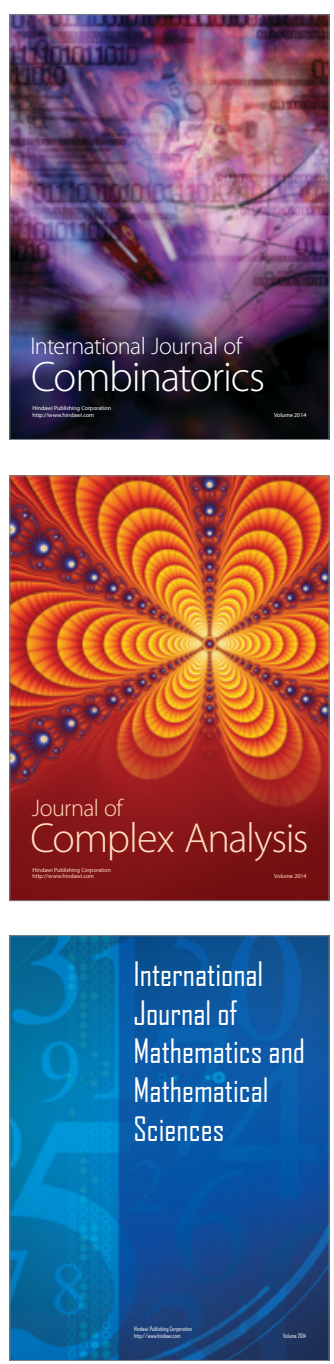


Submit your manuscripts at http://www.hindawi.com
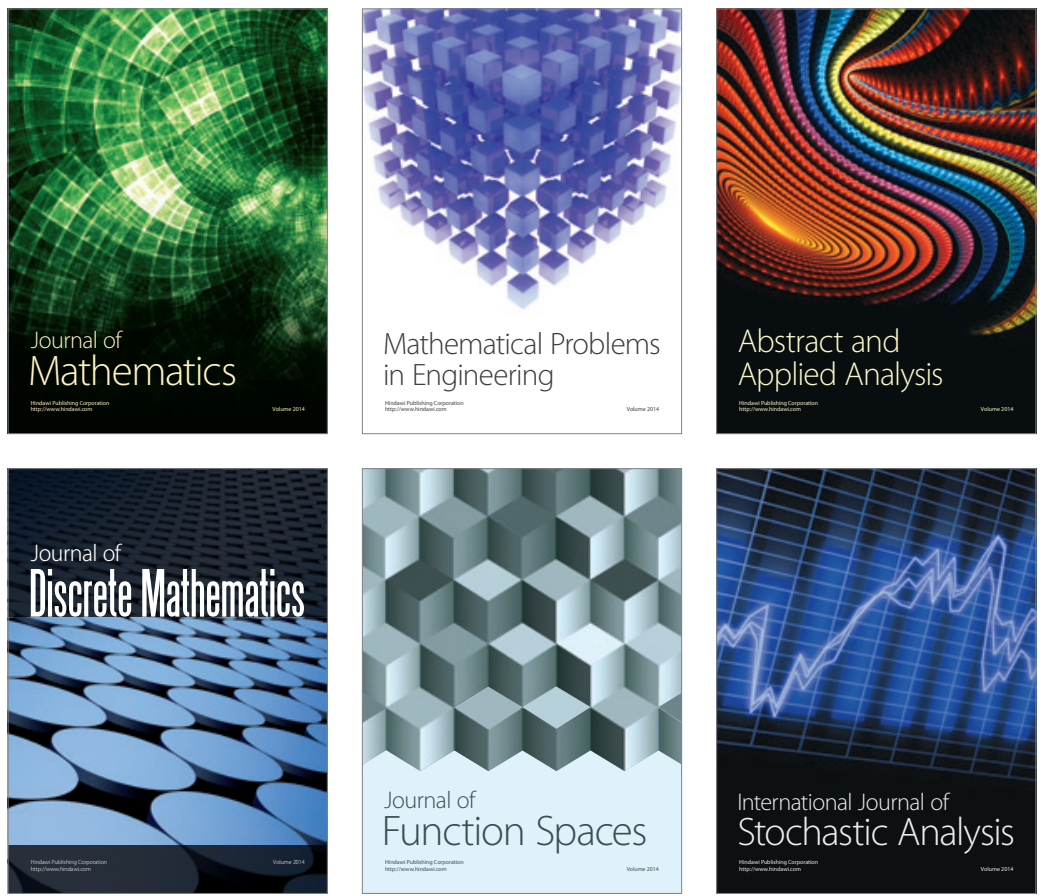

Journal of

Function Spaces

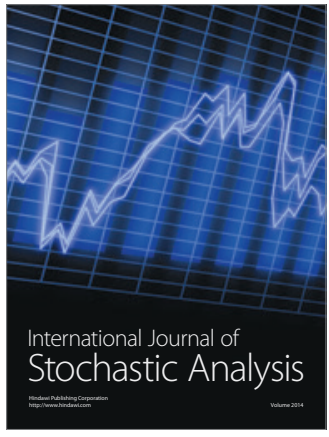

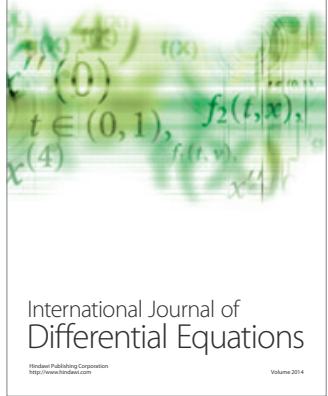
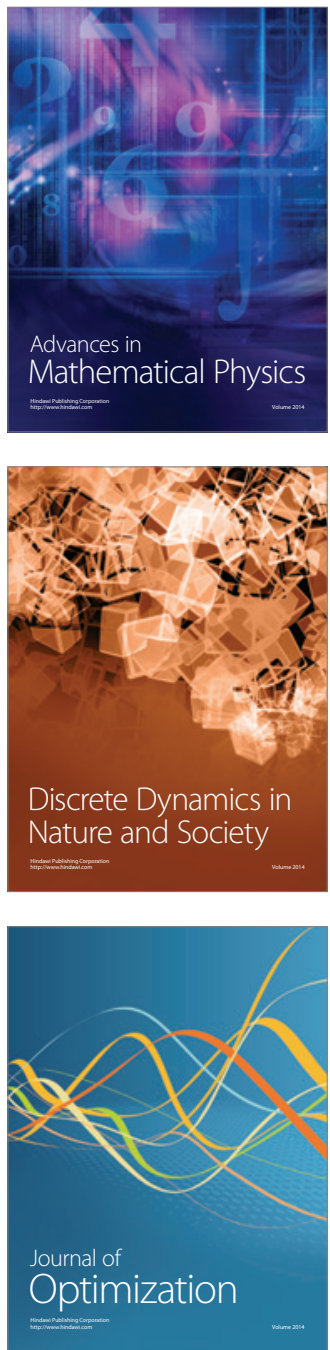